\title{
THE STRUCTURE OF MEASURABLE MAPPINGS ON METRIC SPACES
}

\author{
ANDRZEJ WISNIEWSKI
}

(Communicated by Andrew Bruckner)

\begin{abstract}
The purpose of this paper is to investigate the conditions under which every measurable mapping on a metric space $X$ with the measure $\mu$ is a limit of a sequence of continuous mappings, with respect to the convergence $\mu$-almost everywhere.
\end{abstract}

\section{INTRODUCTION}

It is well known that if $f$ is a function on a finite interval which is measurable with respect to the Lebesgue measure, then $f$ is the limit almost everywhere of a sequence of continuous functions (see, e.g., [4, Theorem 5, p. 104]). This paper is devoted to the generalization of the above fact.

Let $X$ and $Y$ be metric spaces. Denote by $\mathfrak{B}(X)$ and $\mathfrak{B}(Y)$ respectively the Borel $\sigma$-algebras on these spaces. Let $\mu$ be a finite Borel measure on $X$. By $\mathfrak{B}_{\mu}(X)$ we shall denote the completion in the measure $\mu$ of the $\sigma$-algebra $\mathfrak{B}(X)$. A mapping $f$ from $X$ into $Y$ is called $\mu$-measurable if it is measurable with respect to $\left(\mathfrak{B}_{\mu}(X), \mathfrak{B}(Y)\right)$. The simplest example of a $\mu$-measurable mapping is a continuous mapping from $X$ into $Y$.

In $[3$, p. 544] Gihman and Skorohod proved the theorem which shows the connection between continuous and $\mu$-measurable mappings on Hilbert spaces. Namely, they proved that if $X$ and $Y$ are separable Hilbert spaces and $\mu$ is a probability Borel measure on $X$, then for every $\mu$-measurable mapping $f$ from $X$ into $Y$ there exists a sequence of $\left\{f_{n}\right\}$ of continuous mappings from $X$ into $Y$ such that $f_{n} \rightarrow f \mu$-a.e.

In the present paper we shall consider the following problem: is the above theorem of Gihman and Skorohod true for arbitrary metric spaces $X$ and $Y$ and an arbitrary finite Borel measure $\mu$ on $X$ ?

It is easy to see that in general such an extension of this theorem needs not always be true, even under the additional assumption that $X$ and $Y$ are separable and complete metric spaces.

Received by the editors December 2, 1992.

1991 Mathematics Subject Classification. Primary 28C15, 60B05; Secondary 28A20, 28C20, 60B11.

Key words and phrases. Metric spaces, Borel measures, Borel mappings, measurable mappings, continuous mappings. 
For example, if $X=[0,1]$ with the usual metric and the Lebesgue measure and $Y=\{0,1\}$ with the discrete metric, then only the constant functions 0 and 1 are continuous from $X$ to $Y$, while the set of measurable functions consists of all functions $\chi_{A}$, where $A$ is a $\mu$-measurable set.

In this paper we shall give one of the affirmative answers of the stated problem. First we show that this problem has a positive solution if $X$ is an arbitrary metric space and $Y=R$ is the real line (Theorem 1). Then we extend this result to the case where $Y$ is a separable Banach space with the approximation property (Theorem 2 ).

\section{MAIN ResUlts}

Theorem 1. Let $\mu$ be a finite Borel measure on a metric space $X$. If $f$ is a $\mu$-measurable mapping from $X$ into a real line $R$, then there exists a sequence $\left\{f_{n}\right\}$ of continuous mappings from $X$ into $R$ such that $f_{n} \rightarrow f \mu$-a.e.

Proof. To prove the theorem it suffices to show that for any $\varepsilon>0$ and $\rho>0$ there exists a continuous mapping $g: X \rightarrow R$ such that

$$
\mu\{x:|f(x)-g(x)|>\varepsilon\}<\rho .
$$

Indeed, if this is true, then choosing the sequences $\varepsilon \rightarrow 0$ and $\rho \rightarrow 0$ we can construct a sequence of continuous mappings from $X$ into $R$ which is convergent in the measure $\mu$ to $f$, and from this sequence we may choose a subsequence which is convergent to $f \mu$-almost everywhere.

Therefore, let $\varepsilon>0$ and $\rho>0$ be fixed. We must construct a continuous mapping $g: X \rightarrow R$ which satisfies (1). From Lusin's theorem [6, Theorem 21.4] there exists a closed subset $D$ of $X$ such that $\mu(X-D)<\rho$ and the restriction of $f$ to $D$ is continuous. Denote by $\bar{g}$ this restriction, i.e., $\bar{g}=\left.f\right|_{D}$. Thus $\bar{g}$ is a continuous mapping from $D$ into $R$. Since $D$ is a closed subset of $X$, by virtue of Tietze's theorem [5, p. 115] we can extend the mapping $\bar{g}$ to a mapping which is continuous on the whole space $X$. This means that there is a continuous mapping $g: X \rightarrow R$ such that

$$
\{x:|f(x)-g(x)|>\varepsilon\} \subset X-D .
$$

In fact, if the inclusion is not true, then there exists $x \in D$ such that $\mid f(x)-$ $g(x) \mid>\varepsilon$. But on the set $D$ the mappings $f$ and $g$ are equal. Hence $f(x)=$ $g(x)$, which contradicts the above inequality.

From the inclusion (2) and the fact that $\mu(X-D)<\rho$ we obtain (1). The theorem is thus proved.

Now we prove the main result of this paper which shows that Theorem 1 is true if the range of a mapping $f$ is a separable Banach space with the approximation property.

A Banach space $Y$ is said to be a space with the approximation property if for every compact subset $K$ of $Y$ and every $\varepsilon>0$ there exists a finitedimensional continuous linear operator $T$ on $Y$ such that $\|T y-y\|<\varepsilon$ for any $y \in K$. It is easy to see that each Banach space with the Schauder basis has the approximation property (see [1, p. 514]).

Theorem 2. Let $\mu$ be a finite Borel measure on a metric space $X$, and let $Y$ be a separable Banach space with the approximation property. If $f$ is a $\mu$ - 
measurable mapping from $X$ into $Y$, then there exists a sequence $\left\{f_{n}\right\}$ of continuous mappings from $X$ into $Y$ such that $f_{n} \rightarrow f \mu$-a.e.

Proof. Similarly as in the proof of Theorem 1 it is enough to show that for any $\varepsilon>0$ and $\rho>0$ there exists a continuous mapping $g: X \rightarrow Y$ such that

$$
\mu\{x:\|f(x)-g(x)\|>\varepsilon\}<\rho .
$$

Let $\nu$ denote a finite Borel measure on $Y$ given by the formula $\nu(B)=$ $\mu\left(f^{-1}(B)\right)$ for every Borel subset $B$ of $Y$. Since each finite Borel measure on $Y$ is tight (see [2, Theorem 1.4]), there exists a compact subset $K$ of $Y$ such that $\nu(Y-K)<\rho / 2$. Put $K^{\prime}=f^{-1}(K)$. Then

$$
\mu\left(X-K^{\prime}\right)<\rho / 2 .
$$

Now, since $Y$ is a Banach space with the approximation property, there exists a finite-dimensional operator $T$ on $Y$ such that $\|y-T y\|<\varepsilon / 2$ for any $y \in K$. Hence

$$
\|f(x)-T(f(x))\|<\varepsilon / 2 \text { for any } x \in K^{\prime} .
$$

It is well known that each finite-dimensional operator on $T$ can be represented in the form $T y=\sum_{k=1}^{m} f_{k}(y) y_{k}$, where $f_{1}, \ldots, f_{m} \in Y^{*}\left(Y^{*}\right.$ denotes the dual space of $Y$ ) and $\left\{y_{1}, \ldots, y_{m}\right\}$ is a basis of $T(y)$ with $\left\|y_{k}\right\|=1$ for $k=1, \ldots, m$ (see $[1$, p. 492]).

Taking into account this representation, we can write inequality (5) in the form

$$
\left\|f(x)-\sum_{k=1}^{m} f_{k}(f(x)) y_{k}\right\|<\varepsilon / 2 \quad \text { for any } x \in K^{\prime} .
$$

Hence, using (4), we infer that

$$
\mu\left\{x:\left\|f(x)-\sum_{k=1}^{m} f_{k}(f(x)) y_{k}\right\|>\varepsilon / 2\right\} \leq \mu\left(X-K^{\prime}\right) \leq \rho / 2 .
$$

For every $k=1, \ldots, m$ the function $g_{k}: X \rightarrow R$ defined by the formula $g_{k}(x)=f_{k}(f(x))$ is a $\mu$-measurable mapping from $X$ into $R$. Then, in view of Theorem 1 , we see that for every $k=1, \ldots, m$ there exists a sequence $\left\{g_{n}^{(k)}\right\}$ of continuous mappings from $X$ into $R$ such that $g_{n}^{(k)} \rightarrow g_{k}($ as $n \rightarrow \infty)$ $\mu$-a.e.

Hence for every $k=1, \ldots, m$ there exists $n_{k}>0$ such that

$$
\mu\left\{x:\left|g_{k}(x)-g_{n_{k}}^{(k)}(x)\right|>\varepsilon / 2 m\right\}<\rho / 2 m .
$$

Let $g(x)=\sum_{k=1}^{m} g_{n_{k}}^{(k)}(x) y_{k}$. Then $g$ is a continuous mapping from $X$ into 
$Y$. Moreover, from (6) and (7) we have

$$
\begin{aligned}
& \mu\{x:\|f(x)-g(x)\|>\varepsilon\}=\mu\left\{x:\left\|f(x)-\sum_{k=1}^{m} g_{n_{k}}^{(k)}(x) y_{k}\right\|>\varepsilon\right\} \\
& \leq \mu\left\{x:\left\|f(x)-\sum_{k=1}^{m} f_{k}(f(x)) y_{k}\right\|>\varepsilon / 2\right\} \\
&+\mu\left\{x:\left\|\sum_{k=1}^{m} f_{k}(f(x)) y_{k}-\sum_{k=1}^{m} g_{n_{k}}^{(k)}(x) y_{k}\right\|>\varepsilon / 2\right\} \\
& \leq \rho / 2+\sum_{k=1}^{m} \mu\left\{x:\left|g_{k}(x)-g_{n_{k}}^{(k)}(x)\right|>\varepsilon / 2 m\right\} \\
& \leq \rho / 2+m \cdot \rho / 2 m=\rho .
\end{aligned}
$$

This completes the proof of the theorem.

\section{REFERENCES}

1. G. P. Akilov and L. V. Kantorovic, Functional analysis, Nauka, Moscow, 1977.

2. P. Billingsley, Convergence of probability measures, Wiley, New York, 1968.

3. I. I. Gihman and A. V. Skorohod, The theory of stochastic processes. I, Springer-Verlag, Berlin, Heidelberg, and New York, 1974.

4. S. Hartman and J. Mikusinski, The theory of Lebesgue measure and integration, Pergamon Press, Oxford, London, and New York, 1961.

5. K. Jänich, Topology, Springer-Verlag, New York, Berlin, Heidelberg, and New York, 1980.

6. M. E. Munroe, Introduction of measure and integration, Addison-Wesley, Cambridge, 1953.

Institute of Mathematics, Szczecin University, Ul. Wielkopolska 15, 70-451 Szczecin, Poland 